\title{
Benchmark on Human Simulation Tools: A Transdisciplinary Approach
}

\author{
Fabio GRANDI ${ }^{\mathrm{a}}$, Sara CAVALLARO ${ }^{\mathrm{a}}$, Margherita PERUZZINI ${ }^{\mathrm{a}, 1}$, Roberto \\ RAFFAELI ${ }^{\mathrm{b}}$ and Marcello PELLICCIARI ${ }^{\mathrm{b}}$ \\ a Department of Engineering "Enzo Ferrari", University of Modena and Reggio \\ Emilia, Modena \\ ${ }^{\mathrm{b}}$ Department of Sciences and Methods for Engineering, University of Modena and \\ Reggio Emilia
}

\begin{abstract}
Nowadays companies have to face a competitive market that requires small volumes with a high level of customisations. In this context, assembly quality and timeliness is crucial. To guarantee flexibility and personalization, manual operations still have a crucial role for a lot of manufacturing sectors, so that workers' conditions and ergonomics are important factors to achieve a better product quality and overall cost reduction. Ergonomics evaluation in manufacturing is a challenging and expensive activity that requires a transdisciplinary approach, to merge technical and social sciences to finally have a consolidated and reliable evaluation. This paper compared two digital human simulations tools offered by Siemens Tecnomatix: Jack and Process Simulate. They were applied on the same industrial case study, concerning the hood assembly of an agricultural machine, comparing results on ergonomics reports and usage time. Results confirmed the advantage of adopting a digital approach to predict the human effort and ergonomic risk related to a series of tasks. At the same time, they showed the major strengths and weaknesses of the two analysed tools and defined how they can be successfully adopted by companies. The paper finally provided guidelines to drive companies in choosing the best tool according to their needs.
\end{abstract}

Keywords. digital manufacturing, digital human simulations, transdisciplinary engineering, ergonomics, human-centered design.

\section{Introduction}

In the new industrial 4.0 and digital era, a fundamental role is played by factory ergonomics for the optimization of working conditions [1]; safeguarding the health of operators has become an increasingly crucial issue for most companies whose production process is based on manual production and assembly activities [2]. Generally, ergonomic assessment requires a great effort in terms of time and costs and a certain level of expertise during the analysis. Moreover, in traditional approaches, ergonomic analysis is carried out at the shop-floor, when the plant is already created and changes to workstations are challenging and expensive [3]. Consequently, an urgent need for companies is to improve and speed up the ergonomic analysis carried out on plants and production lines [4].

\footnotetext{
${ }^{1}$ Corresponding Author, Mail: margherita.peruzzini@unimore.it.
} 
Assembly is still one of those activities in smart factories carried out by humans, especially to satisfy production flexibility and personalization on small batches. Assembly lines of manufacturing industries are very complex to design, because production processes are affected by several variables, such as technological, environmental, logistics and ergonomics. In particular, ergonomic assessments are often barely considered during the design phase, due to the complexity; this implies the need to correct the design later when ergonomic issues occur during the production phase, with high-cost of redesign [5]. Moreover, the extreme pressure for companies to achieve their businesses to be competitive in their markets could have a negative impact on the workers' mental workload and well-being. In the manufacturing sector, the effective implementation of ergonomics aspects in processes has been proven to decrease costs related to musculoskeletal disorders (MSD), extratime hours, medical care or fines for risk-events [6]. In addition, according to the literature, harmful ergonomic risk factors affect not only human well-being on work but also human performance, decreasing the product quality. For this reason, companies should be convinced that incorporation of an ergonomic approach in the production system would be profitable in the short and long term [7].

Digitization is one of the main pillars of the Industry 4.0 revolution; digital tools can support the simulation of industrial processes in order to optimize the production process and to enhance workers' conditions [8]. Transdisciplinary engineering (TE) methods can be successfully applied to solve complex problems linked to Digital Manufacturing (DM) [9]. To include people in the production process, DM tools help engineers and technicians to predict critical working conditions, bridging the gap between technical and social sciences [10]. The aim of this paper is to critically compare two of the most used Digital Human Simulation (DHS) software toolkits, Jack and Process Simulate (PS), in order to drive companies in the adoption of these tools in an effective way, through performance indicators.

\section{Research background}

Nowadays companies have to adopt digitization to be in line with market trends; this fact can highly benefit also ergonomics, moving from a corrective to a predictive approach [11]. In detail, the available digital tools include sophisticated digital human models, regional anthropometric databases, and the latest ergonomic methods. Digital simulations are used to analyze humans' working conditions in different contexts, from driving to manufacturing, supporting the evaluation of the user comfort and the workstation designs to assure the proper ergonomic conditions for the workers. Nevertheless, these human simulations are generally carried out by human factors engineers or ergonomists, using dedicated tools, whereas the production simulations are carried out by production engineers [12]. Diversely, a real benefit could be achieved by merging these two activities. Indeed, the adoption of virtual mannequins into process simulation can help designers and engineering to consider the human factors from the early design phases, to produce more human-centered vehicles, equipment, assembly lines, manufacturing plants, interfaces, and interactive systems in general [13]. The scientific literature shows numerous examples of successful applications of DHS in the manufacturing context, under the name of DHS systems [14-16]. In particular, DHS are widespread for assembly task evaluation, in which a knowledge-based decision support tool could facilitate the optimization of workflow [17], from a human-centered 
perspective. A preventive ergonomic approach is useful to drastically reduce time-tomarket of manufactured products, and consequently their costs. Indeed, 70 to 80 percent of the life-cycle costs of a product are determined by decisions taken by designers during the early stages of the design process. Therefore it is important to apply ergonomic assessment as early and as accurately as possible [18].

For these purposes, several ergonomic analysis methods and techniques have been recently developed within the DHS softwares, to support engineers. Two DHS softwares have been identified, Jack and PS by Siemens, to be compared for industrial applications. Jack is a specific module dedicated to comfort analysis; it is probably one of the most popular and currently used DHS software as demonstrated also by Mühlstedt [19]. On the contrary, PS is integrated in the Siemens PLM offer and allows starting from the production digital scenario and implementing one or more workstations. Each software has its strengths and weaknesses. Therefore it is not only important to take the analysis with these softwares, but also to be able to interpret and apply their outputs [20].

\section{The approach for system comparison}

A transdisciplinary approach starts from a real-world problem and connects complex and interdependent social and engineering issues [21]: DHS is a key technology that allows human factors to be addressed during both the digitalized product design and productionplanning phases. The proposed approach aims to compare the above-mentioned DHS softwares in order to recognize advantages and weaknesses in a real case study. Both of them allow the creation of process simulations and ergonomic analysis, improving decision-making capacity of engineers and production planners as required by a transdisciplinary approach [21]. The comparison could be useful for companies to identify the best solution that fits their needs, in order to solve ergonomic issues. The study has been developed in collaboration with a tractor manufacturer, focusing on the real activities carried out by workers on the production plant.

\subsection{Comparison methodology}

The proposed methodology was conceived in order to give a holistic evaluation of the two systems under evaluation. For this purpose, a set of performance indicators has been defined in order to evaluate both qualitative and quantitative aspects and to finally generate the overall ergonomic report. The proposed comparison is based on subjective and objective indicators as described in Table 1. The first section describes the selected indicators adopted for subjective assessment, as expressed by the experts involved in the study. The second and third section list two classes of objective indicators, respectively about time performance and ergonomic assessment by a set of selected metrics.

Subjective indicators consider the effort to digitize the assembly task sequence, to carry out the simulation until the analysis of the ergonomics results. The evaluation was carried out by experts using the 5-point Likert scale taking into account a set of software features in order to give a qualitative performance assessment. In particular, the identified indicators are measured considering:

- Easiness of scene creation: ease of importing virtual models in the scene;

- Easiness of scene navigation: usability of scene navigation commands;

- Easiness of object manipulation: simplicity of moving parts in the virtual scene; 
- Easiness of joint manipulation: straightforwardness in positioning the virtual mannequin in a realistic way.

Objective indicators refer to time performance and accuracy of the ergonomic assessment. About time performance, indicators consider the time to accomplish the main tasks:

- Scene creation;

- Simulation implementation;

- Ergonomic report creation;

- Ergonomic analysis.

Accuracy of the ergonomic assessment is evaluated considering the score obtained using internationally-recognized ergonomic methods, suitable to assess the risk to develop muskulo-skeletal disorders, according to the specific analysis to be performed. The selected indicators are as follows:

- Ovako Working posture Analysing System (OWAS): it is a concise system for postural assessment that provides an holistic evaluation of workers physical effort. Each posture is designated by a 4-digit code that depends on the classification of the current posture with respect to four predefined levels of danger. It takes into account the postures assumed by the worker and evaluates each posture according to the position of the back, arms, legs, and to the weight lifted;

- Rapid Upper-Limb Assessment (RULA): this method uses a checklist in which each body part is carefully evaluated, according to a set of predefined limit positions. It evaluates the overall comfort of a working posture analyzing the position of the trunk, neck, legs, wrist, upper and lower limbs as well as the presence of loads. A general score is assigned to each posture, which indicates the necessity of changing the workstation layout, in order to reduce the risk of potential physical disorders to the operator;

- Ergonomic Assessment Work-Sheet (EAWS): it is a tool for the holistic evaluation of physical workloads, based on standardised process languages such as Methods-Time Measurement (MTM) and Universal Analyzing System (UAS). EAWS focuses especially on existing CEN and ISO standards, evaluating working postures, action forces, manual materials handling and repetitive loads of the upper limbs. It evaluates the physical effort with three zones (traffic light scheme) rating system;

- Cumulative low Back Loading (CBL): this tool evaluates the spinal forces acting on a workers's lower back, under any posture and loading condition. It calculates the low back compression, anterior/posterior shear and lateral shear at L4/L5 vertebral joint and shows if loads exceed NIOSH threshold limit values or expose workers to an increased risk of low back injury forces.

Ergonomic indicators' scores extracted from the two softwares can be also compared with a video-based ergonomic analysis carried out by experts or by specific, dedicated software tools (e.g. motion capture systems).

\section{The industrial case study}

The industrial case study is based on the assembly process design of a medium-sized tractor hood. In the company, this type of process is accomplished by a completely manual procedure. The use of DHS was useful to design the process taking advantage of the digital support. Both tools were used and compared according to the proposed 
methodology. After that, the assembly line was really implemented and real workers were video-recorded to carry out the traditional, video-based ergonomic analysis by experts. Four experts were involved in the study; they judged the tool usage by the proposed methodology and carried out a video-based traditional analysis for the evaluation of the ergonomic indicators.

Table 1. Comparative indicators.

\begin{tabular}{lll}
\hline & Indicators & Unit of measurement \\
\hline \multirow{2}{*}{ Subjective } & Easiness of scene creation & $1-5$ Likert scale \\
\cline { 2 - 3 } & Easiness of scene navigation & $1-5$ Likert scale \\
\cline { 2 - 3 } & Easiness of object manipulation & $1-5$ Likert scale \\
\cline { 2 - 3 } $\begin{array}{l}\text { Time } \\
\text { performance }\end{array}$ & Easiness of joint manipulation & $1-5$ Likert scale \\
\cline { 2 - 3 } & Time for scene creation & Hours \\
\cline { 2 - 3 } & Time for simulation implementation & Hours \\
\cline { 2 - 3 } $\begin{array}{l}\text { Accuracy of } \\
\text { ergonomic } \\
\text { assessment }\end{array}$ & Time for ergonomic report creation & Hours \\
\cline { 2 - 3 } & Time for ergonomic report analysis & Hours \\
\cline { 2 - 3 } & EAWS & Level of risk (green, yellow, red $)$ \\
\cline { 2 - 3 } & CBL & Level of risk $(0-25,26-50,>50)$ \\
\hline
\end{tabular}

\subsection{Case study description}

The case study was originally analyzed together with the company engineering team, which highlighted the necessity to design the assembly sequence by acting both on the product and the workstation, due to the possible operator's difficulties in reaching some parts and viewing the hood fixing points. Figure 1 shows the digitization of the workstation as originally designed by the company.

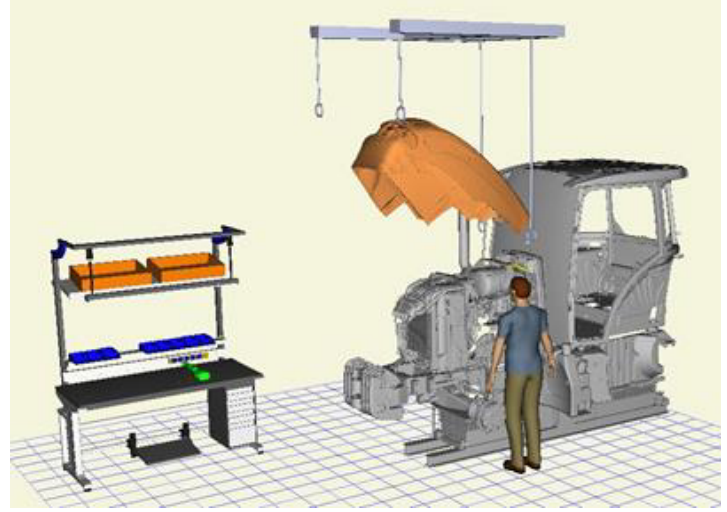

Figure 1. Digitization of the workstation as originally designed. 
The as-is task sequence of the hood assembly, as considered in this study, is madeup of 10 steps and described in Table 2.

Table 2. Use case tasks.

\begin{tabular}{cl}
\hline $\mathbf{N}^{\circ}$ of task & \\
\hline 1. & Pick up the hood from the conveyor with a hoist \\
\hline 2. & Lower the hood with the hoist onto the tractor body \\
\hline 3. & $\begin{array}{l}\text { Guide the hood with hands to center the four threaded pins in the holes of the hood support } \\
\text { bracket }\end{array}$ \\
\hline 4. & Pick up n.2 bolts from the box storage \\
\hline 5. & Start the bolts manually \\
\hline 6. & Pick up n.2 nuts from the box storage \\
\hline 7. & Start the nuts manually \\
\hline 8. & Pick up electric screwdriver from the trolley \\
\hline 9. & Tighten the nuts with the electric screwdriver \\
\hline 10. & Place the electric screwdriver on the trolley \\
\hline
\end{tabular}

Moreover, precise indications were submitted regarding boundary conditions such as the maximum hood's opening angle $\left(50^{\circ}\right)$, the hood positioning direction (the hood must be collected from the right side of the tractor body) and the number of operators (one operator per side). In order to correctly implement the simulation, objects' weights need to be simulated to properly evaluate the physical effort of operators. The weights of the parts to be handled are: the tractor hood $(50 \mathrm{~kg})$, the two nuts $(0,03 \mathrm{~kg})$, the 2 screws $(0,04 \mathrm{~kg})$ and the electric screwdriver $(4 \mathrm{~kg})$. In addition, a report about loads (forces and momentums) that workers have to apply during the assembly was reported by the engineering team.

\subsection{Case study simulation}

The case study was virtually recreated in the two simulation environments, as shown in Figure 2. Regarding Jack, the simulation was implemented with the use of the Task Simulation Builder (TSB) plugin, in which the user could define the workers' tasks step by step. Similarly, the worker task sequence was created in PS using the same pattern included in the main software.

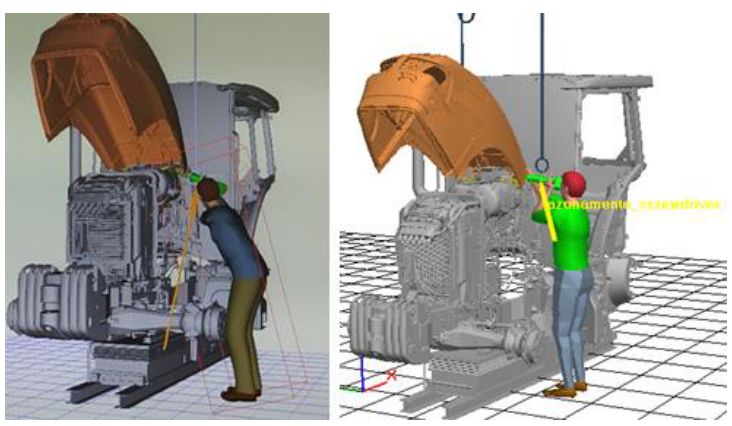

Figure 2. Simulation of a worker in Jack (left) and PS (right) environment. 


\section{Results from the comparative study}

\subsection{Subjective evaluation}

Results from the subjective evaluation are reported in Figure 3 as average scores on the four experts involved, using the 5-point Likert scale. Regarding the scene creation, the operations to load 3D CAD models or create the mannequins do not require particular skills, so this highlights that Jack is more intuitive and easy to use compared to PS. On the contrary, the latter seems to be more direct about scene navigation, as shown in Figure 3 , because Jack requires a combination of keyboard and mouse commands to rotate and zoom the virtual environment. Furthermore, the object manipulation is more accurate and straightforward on PS than Jack. Instead, the manikin joint manipulation is very similar on the two softwares and there is not particular criticality.

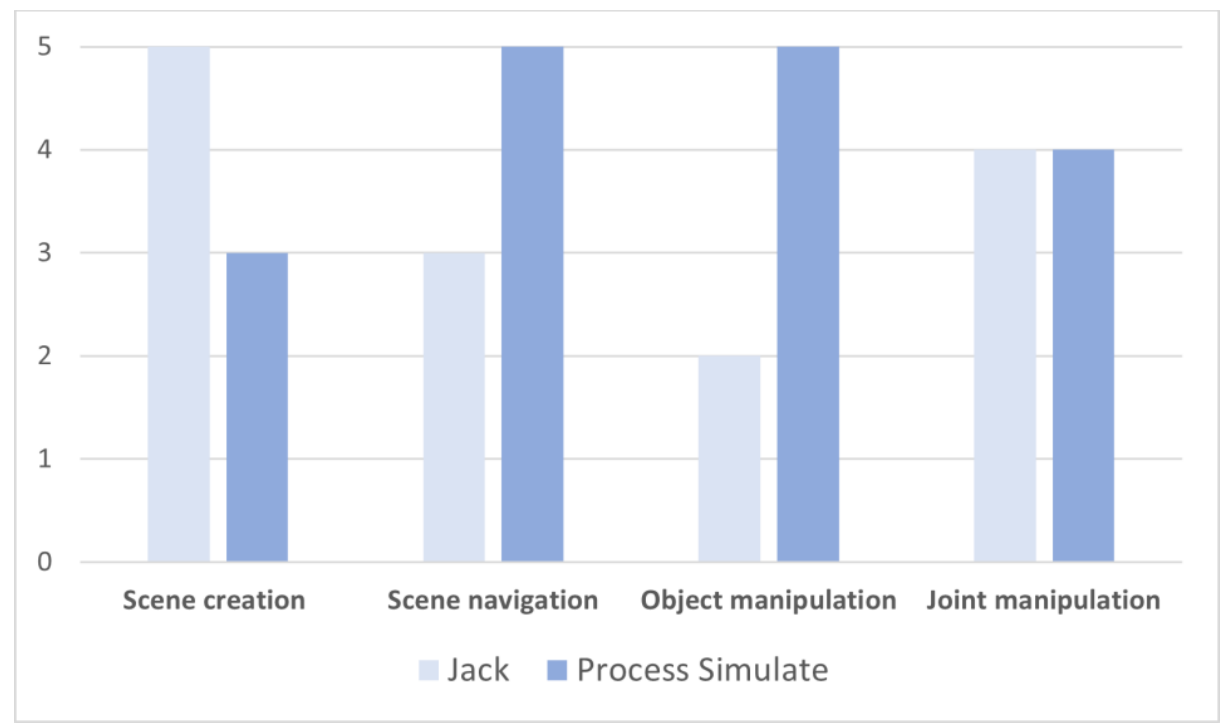

Figure 3 . Subjective assessment.

\subsection{Objective evaluation}

Objective evaluation included the analysis of time performance and the accuracy of the ergonomic assessment. About time performance, Table 3 synthesizes the comparison between Jack and PS, as average values obtained by all experts. Results demonstrated that analysis in Jack is more effective for scene creation ( $-33 \%$ on average), but it is globally more time consuming $(+70 \%$ on average), mainly due to the higher time requested to create the ergonomic reports and their analysis. In both cases, the simulation implementation time is comparable between the two softwares. As far as the accuracy of the ergonomic assessment, the scores extracted from the two softwares analysis on the whole task sequence and the video-based ergonomic analysis by experts were compared. In the interest of brevity, values indicated in Table 4 take into account the overall scores on the entire process. For OWAS and RULA, the average score on the entire sequence was considered. 
Table 3. Comparison on time performance indicators.

\begin{tabular}{lcc}
\hline Tasks & Jack (h) & PS (h) \\
\hline Scene creation & 2 & 3 \\
\hline Simulation implementation & 6 & 6 \\
\hline Creation of ergonomic reports & 10 & 3 \\
\hline Ergonomic report analysis & 8 & 3 \\
\hline Total & 26 & 15 \\
\hline
\end{tabular}

Table 4. Comparison on accuracy of ergonomic assessment (*average values of the entire task sequence)

\begin{tabular}{lccc}
\hline Ergonomic Index & On field assessment & Jack & PS \\
\hline OWAS* & yellow risk & yellow risk & yellow risk \\
\hline RULA* & 4,8 & 4,7 & 4,5 \\
\hline EAWS & 37,3 & 43,0 & 35,1 \\
\hline CBL [kNs] & 4214,4 & 3958,3 & 4365,9 \\
\hline
\end{tabular}

From these results, we can state that:

- OWAS comparison does not highlight any significant differences in terms of risk level between Jack, PS and expert video-based ergonomic analysis, considering the average score during the entire simulation. Some minimal differences in the score of the single postures are revealed. The application on the use case spotted an issue in PS that badly evaluated the task in which operators apply forces.

- $\quad$ RULA comparison demonstrated a good match by scores obtained by Jack, PS and expert video-based ergonomic analysis as well. Only notable difference is the risk band associated with the Apply Force task that is part of the nut tightening phase; in fact, PS underestimate the risk respect Jack in this kind of tasks;

- EAWS comparison underlined that PS is a more reliable tool for this calculation, providing an overall score closer to the experts evaluation. This could be due to the fact that this index is automatically calculated only in PS software but not in Jack, where is required a post processing of data postures in an external dedicated excel checklist;

- Finally, the CBL comparison observed that the cumulative compression value on L4/L5 (considered in a shift of 8 working hours) from Jack and PS simulations have given approximately the same results, that were far below the NIOSH limit. We could say that Jack mildly underestimates the risk, but we did not measure this risk on the field.

To sum up, the main strengths of both systems are listed in Table 5, to help companies to choose the system that better satisfies their needs. 
Table 5. Points of strengths of Jack and PS.

\begin{tabular}{ll}
\hline Software & \multicolumn{1}{c}{ Points of strengths } \\
\hline \multirow{2}{*}{ Jack } & easier and faster scene creation \\
\cline { 2 - 2 } & more reliable results on apply force tasks in OWAS an RULA evaluation \\
\hline \multirow{3}{*}{ PS } & more intuitive object manipulation and scene navigation \\
\cline { 2 - 2 } & faster creation and analysis of ergonomic assessment report \\
\cline { 2 - 2 } & automatic and more accurate EAWS evaluation \\
\hline
\end{tabular}

\section{Conclusions}

The paper investigated the application of DHS in manufacturing tasks to support the design of human-centric workstations, considering two software toolkits, Jack and Process Simulate from Siemens. In particular, the paper proposed a methodology to compare any type of DHS softwares, defining a set of subjective and objective indicators. Ergonomic problems investigated by different analysis tools benefit from a transdisciplinary approach that merges the engineering point of view with the social sphere, considering the well-being of the operators [22][23]. The aim of the research is to guide companies in the selection of the most suitable software that fits their needs, encouraging the application of transdisciplinary tools. The comparison study demonstrated that Jack is probably the best choice to optimize the scene creation effort and ease of use, and to execute formative analysis on brief, static postures. Moreover, Jack makes us obtain more reliable results in case of huge force applications. On the contrary, PS allows an easier object manipulation and virtual scene navigation compared to Jack. Furthermore, PS is more suitable to carry out an overall ergonomic evaluation considering an entire process, providing a more detailed ergonomic report and allowing to save time during the ergonomic analysis itself. In particular, the EAWS evaluation in PS is immediate, using a dedicated plugin, that provides accurate results. In brief, Jack could be effectively used for quick static posture evaluation, instead PS is advisable for ergonomic studies with dynamic simulation.

The main limitations of the study are due to the limited number and complexity of the simulated tasks of the case study. For this reason, the comparison could be extended to other application fields, including both assembly and maintenance operations.

\section{References}

[1] M. Gášová, M. Gašo and A. Štefánik, Advanced industrial tools of ergonomics based on Industry 4.0 concept, Procedia Engineering, 2017, Vol. 192, pp. 219-224.

[2] Y. Cohen, M. Faccio, F.-G. Galizia, C. Mora and F. Pilati, Assembly system configuration through Industry 4.0 principles: the expected change in the actual paradigms. IFAC-PapersOnLine, Vol. 50(1), pp. 14958-14963. 2017,

[3] M. Peruzzini, S. Carassai and M. Pellicciari, The benefits of human-centred design in industrial practices: re-design of workstations in pipe industry. Procedia Manufacturing, 2017, Vol. 11, pp. 1247-1254.

[4] L. Hanson, The Need for Faster and More Consistent Digital Human Modeling Software Tools, Advances in Transdisciplinary Engineering, Vol. 11, 2020, pp. 299-310. 
[5] F. Caputo, A. Greco, M. Fera and R. Macchiaroli, Workplace design ergonomic validation based on multiple human factors assessment methods and simulation. Production \& Manufacturing Research, 2019, Vol. 7(1), pp. 195-222.

[6] M.-F. Brito, A.-L. Ramos, P. Carneiro and M.-A. Gonçalves, Ergonomic Analysis in Lean Manufacturing and Industry 4.0 - A Systematic Review.in A.C. Alves et al. (eds.) Lean Engineering for Global Development, Springer Nature Switzerland, 2019, pp. 95-127.

[7] M. Zare, M. Croq, F. Hossein-Arabi, R. Brunet and Y. Roquelaure, Does ergonomics improve product quality and reduce costs? A review article. Human Factors and Ergonomics in Manufacturing \& Service Industries, 2016, Vol. 26(2), pp. 205-223.

[8] G. Chryssolouris, D. Mavrikios, N. Papakostas, D. Mourtzis, G. Michalos and K. Georgoulias, Digital manufacturing: history, perspectives, and outlook. Proceedings of the Institution of Mechanical Engineers, Part B: Journal of Engineering Manufacture, 2009, Vol. 223(5), pp. 451-462.

[9] E. Rauch, D. T. Matt, C. A. Brown, W. Towner, A. Vickery and S. Santiteerakul, Transfer of industry 4.0 to small and medium sized enterprises. Advances in Transdisciplinary Engineering, 2018, Vol. 4, pp. 63-71.

[10] M. Peruzzini, N. Wognum, C. Bil and J. Stjepandic, Special issue on 'transdisciplinary approaches to digital manufacturing for industry 4.0', International Journal of Computer Integrated Manufacturing, 2020, Vol. 33(4), pp. 321-324.

[11] N. A. Stanton, A. Hedge, K. Brookhuis, E. Salas and H. W. Hendrick, Handbook of human factors and ergonomics methods, CRC press, Boca Raton, 2004.

[12] A. Iriondo Pascual, D. Högberg, A. Syberfeldt, E. Brolin and L. Hanson, Optimizing Ergonomics and Productivity by Connecting Digital Human Modeling and Production Flow Simulation Software. Advances in Transdisciplinary Engineering, 2020, Vol. 13, pp. 193-204.

[13] N. Badler, Virtual humans for animation, ergonomics, and simulation. Proceedings IEEE Nonrigid and Articulated Motion Workshop, 1997, pp. 28-36.

[14] H. Honglun, S. Shouqian and P. Yunhe, Research on virtual human in ergonomic simulation. Computers \& Industrial Engineering, 2007, Vol. 53(2), pp. 350-356.

[15] M. C. Schall Jr, N. B. Fethke and V. Roemig, Digital human modeling in the occupational safety and health process: An application in manufacturing. IISE transactions on occupational ergonomics and human factors, 2018, Vol. 6(2), pp. 64-75.

[16] W. Zhu, X. Fan and Y. Zhang, Applications and research trends of digital human models in the manufacturing industry. Virtual reality \& intelligent hardware, 2019, Vol. 1(6), pp. 558-579.

[17] T.-A. Abdullah, K. Popplewell and C.-J. Page, A review of the support tools for the process of assembly method selection and assembly planning. International Journal of Production Research, 2003, Vol. 41(11), pp. 2391-2410.

[18] M. Saravi, L. Newnes, A. R. Mileham and Y. M. Goh, Estimating cost at the conceptual design stage to optimize design in terms of performance and cost, In: R. Curran et al. (eds.) Collaborative product and service life cycle management for a sustainable world, Springer, London, 2008, pp. 123-130.

[19] J. Mühlstedt, Entwicklung eines Modells dynamisch-muskulärer Arbeitsbeanspruchungen auf Basis digitaler Menschmodelle, PhD thesis, TU Chemnitz, 2012.

[20] P. Polášek, M. Bureš and M. Šimon, Comparison of digital tools for ergonomics in practice. Procedia engineering, 2015, Vol. 100, pp. 1277-1285.

[21] R.W. Scholz and G. Steiner, The real type and ideal type of transdisciplinary processes: part I-theoretical foundations, Sustainability Science, 2015,Vol. 10, No. 4, pp.527-544.

[22] N. Wognum, C. Bil, F. Elgh, M. Peruzzini, J. Stjepandić, W. Verhagen, Transdisciplinary engineering research challenges, Advances in Transdisciplinary Engineering, Vol. 7, 2018, pp. 753-762.

[23] N. Wognum, C. Bil, F. Elgh, M. Peruzzini, J. Stjepandić, W. Verhagen, Transdisciplinary systems engineering: implications, challenges and agenda, International Journal of Agile Systems and Management, 2019, 12(1), pp. 58-89. 